\title{
Pre-treatment systemic immune-inflammation represents a prognostic factor in patients with advanced non-small cell lung cancer
}

\author{
Rossana Berardi ${ }^{1}$, Matteo Santoni ${ }^{1}$, Silvia Rinaldi ${ }^{1}$, Marc Bower ${ }^{2}$, Michela Tiberi ${ }^{3}$, Francesca Morgese ${ }^{1}$, \\ Miriam Caramanti ${ }^{1}$, Agnese Savini ${ }^{1}$, Consuelo Ferrini ${ }^{1}$, Mariangela Torniai ${ }^{1}$, Ilaria Fiordoliva ${ }^{1}$, \\ Thomas Newsom-Davis ${ }^{2}$
}

${ }^{1}$ Clinica Oncologica, Università Politecnica delle Marche, Azienda Ospedaliero-Universitaria Ospedali Riuniti Umberto I, GM Lancisi, G Salesi di Ancona, Italy; ${ }^{2}$ Chelsea \& Westminster Hospital, London, UK; ${ }^{3}$ Chirurgia Toracica, Università Politecnica delle Marche, Azienda OspedalieroUniversitaria Ospedali Riuniti Umberto I, GM Lancisi, G Salesi di Ancona, Italy

Contributions: (I) Conception and design: R Berardi, M Santoni; (II) Administrative support: R Berardi; (III) Provision of study materials or patients: R Berardi, M Santoni, S Rinaldi; (IV) Collection and assembly of data: R Berardi, S Rinaldi, T Newsom-Davis, M Tiberi, C Ferrini; (V) Data analysis and interpretation: R Berardi, M Santoni, S Rinaldi, T Newsom-Davis; (VI) Manuscript writing: All authors; (VII) Final approval of manuscript: All authors.

Correspondence to: Prof. Rossana Berardi. Clinica Oncologica, Università Politecnica delle Marche, Azienda Ospedaliero-Universitaria, Ospedali Riuniti Umberto I, GM Lancisi, G Salesi di Ancona, Via Conca 71, 60126 Ancona, Italy. Email: r.berardi@univpm.it.

\begin{abstract}
Background: Inflammation plays an important role in pathogenesis, development and progression of lung cancer. The aim of the study is to assess the prognostic role of Systemic Immune-Inflammation Index (SII), obtained by analyzing the neutrophil, lymphocyte and platelet counts, and to design prognostic models for patients receiving first-line chemo- or targeted therapy for advanced non-small cell lung cancer (NSCLC).

Methods: We conducted an analysis on 311 patients with advanced NSCLC, treated with first line chemoor targeted therapy till June 2015 at our Institution. Patients were stratified in two groups with SII $\geq 1,270$ (Group A) vs. SII <1,270 (Group B). Progression free survival (PFS) and overall survival (OS) were estimated using Kaplan-Meier method. The best SII cutoff was identified by X-tiles program. A Cox regression model was carried out for univariate and multivariate analyses.

Results: At baseline, 179 patients had SII $\geq 1,270$ (Group A), whilst 132 had lower SII (Group B). The median OS was 12.4 months in Group A and 21.7 months in Group B $(\mathrm{P}<0.001)$, whilst the median PFS was 3.3 and 5.2 months, respectively $(\mathrm{P}=0.029)$. At multivariate analysis, male gender, ECOG-PS $\geq 2$ and SII $>1,270$ were predictors of worst OS, whilst IV tumor stage was only slightly significant $(\mathrm{P}=0.08)$. Otherwise, only wild-type EGFR status and $\mathrm{SII} \geq 1,270$ were independent prognostic factors for worst PFS.

Conclusions: Pre-treatment SII is an independent prognostic factor for patients with advanced NSCLC treated with first-line therapies.
\end{abstract}

Keywords: Lung cancer; platinum-base chemotherapy; prognosis; systemic immune-inflammation index; targeted therapy

Submitted Aug 10, 2018. Accepted for publication Jun 19, 2019.

doi: $10.21037 /$ atm.2019.09.18

View this article at: http://dx.doi.org/10.21037/atm.2019.09.18 


\section{Introduction}

Non-small cell lung cancer (NSCLC) represents approximately the $85-90 \%$ of all lung cancers. It is the leading cause of cancer related death, worldwide, in both sex and its incidence are still increasing (1). Currently, many treatment options are available for these patients. However, their prognosis remains poor, suggesting that reliable bookmakers are strongly required in order to guide treatment decisions and optimize patients' outcome.

Inflammation plays an important role in pathogenesis, development and progression of lung cancer. It has been showed that the most common chronic inflammatory lung diseases, as sarcoidosis or chronic obstructive pulmonary disease (COPD), increase the risk of lung cancer occurrence (2), whilst the chronic use of nonsteroidal antiinflammatory drugs seems to prevent its onset (3).

Inflammatory markers, such as elevated neutrophil count and thrombocytosis, have been associated with the prognosis of NSCLC patients. Indeed, elevated neutrophil count has been correlated with poor outcome in NSCLC patients treated with chemotherapy, with an overall survival (OS) reduction of about 9 months (19.3 vs. 10.2 months) compared to patients with normal neutrophil count (4). In addition, NSCLC patients with preoperative thrombocytosis, platelet-to-lymphocyte ratio (PLR), neutrophil/lymphocyte ratio and prognostic nutritional index showed a significantly shorter disease-free survival (DFS) and OS (5-7). Similarly, elevated platelets count, C-reactive protein and high fibrinogen level were demonstrated as independent poor prognostic factors also in patients with advanced NSCLC $(8,9)$.

Recently, a novel inflammatory parameter, named Systemic Immune-Inflammation Index (SII), obtained by analyzing the neutrophil, lymphocyte and platelet counts, has been investigated as a prognostic factor in patients with hepatocellular carcinoma (HCC). In this study, $\mathrm{Hu}$ and colleagues reported that high SII represented a prognostic indicator of poor outcome in HCC patients after curative tumor resection (10).

In this study, we investigated the prognostic role of Systemic Immune-Inflammation in patients with advanced NSCLC treated with first-line chemo- or targeted therapy.

\section{Methods}

\section{Study population and data collection}

We retrospectively collected data of patients treated with first-line therapy for advanced NSCLC. The study population included adults (age $\geq 18$ years) with histologically or cytologically diagnosis of NSCLC. All patients were treated according to EGFR mutational status with firstline chemotherapy or targeted therapy, at our Institutions between 1st May 2006 and 30th June 2015. Tumor stages were established according to the tumor-node-metastasis (TNM) criteria, using the 7th edition. Patients with IIIB and IV stage were included; patients with IIIA stage, when not eligible for curative surgery, were also included. We excluded patients receiving surgery or radiotherapy, with palliative intent, within 1 month from the start of first-line therapy or presenting factors influencing SII (see below).

First-line therapy was continued until clinical and/or radiological progression or unacceptable adverse events (AEs) or death. Follow-up was performed by physical examination, periodical laboratory analysis and computed tomography (CT) or magnetic resonance imaging (MRI) scans every $8-12$ weeks.

OS was defined as the time from the start of first-line therapy to death. Disease progression was defined according to the RECIST 1.0 criteria (11). Patients with partial or complete remission or presenting stable diseases were considered as responders. Progression free survival (PFS) was defined as the time from the start of first-line therapy to progression or death. Patients without tumour progression or death at data collection time were censored at their last date of evaluation.

Peripheral blood samples were picking up 1 to 7 days before the beginning of first-line therapy. We excluded all patients without available data on pre-treatment neutrophil, lymphocyte and platelet counts, and patients with SII influencing factors, including essential thrombocythemia (ET), chronic myelogenous leukemia (CML), chronic inflammatory diseases, autoimmunity and recent therapy with granulocyte colony stimulating factor (G-CSF) or steroids. Data were collected from electronic medical records and paper charts. Patient's personal data have been secured. Our project has been approved by local Ethics Committee (ID 2019-146) and the work conforms to the provisions of in accordance with the Helsinki Declaration as revised in 2013.

\section{Statistical analysis}

PFS and OS were estimated from Kaplan-Meier curves with Rothman's 95\% confidence intervals (CI). The comparison among groups was performed by the log-rank test. 
Pre-treatment SII was calculated by multiplying the absolute platelet and neutrophil counts and dividing by the absolute lymphocyte count $(\mathrm{SII}=\mathrm{P} \times \mathrm{N} / \mathrm{L})$. Clinicpathological factors potentially correlated with the patients' prognosis were estimated. The list of evaluated factors included age ( $\geq 70 v s .<70$ years), gender, tumor histology [non-adenocarcinoma (non-ADC) vs. ADC], stage, EGFR mutational status, Eastern Cooperative Oncology GroupPerformance Status (ECOG-PS), smoking history and SII. The cut-off that best discriminated between good and poor survival was determined by the X-tile 3.6.1 software (Yale University, New Haven, CT, USA) (12).

Univariate and multivariate Cox regression analyses of potential factors affecting patients' outcome were performed. No-multicollinearity of the grouped co-variates was checked. Significance level at the univariate model for inclusion in the multivariate model was set at $0.2(13,14)$. All other significance levels were set at 0.05 and all $\mathrm{P}$ values were two-sided. Statistical analysis was performed by MedCalc version 11.4.4.0 (MedCalc Software, Broekstraat 52, 9030 Mariakerke, Belgium).

\section{Results}

\section{Patient characteristics}

Out of 311 patients with advanced NSCLC, treated at our institution, 216 were males (69\%). The median age was 68 years (range, 25-86 years). Two hundred and thirty-six patients $(76 \%)$ were current or former smokers. Histology was $\mathrm{ADC}$ in $61 \%$, whilst $22 \%$ had squamous carcinomas and $17 \%$ other tumor histologies. Tumor stage was III in $75(24 \%)$ patients and IV in 236 patients (76\%). Firstline therapy was chemotherapy in 290 patients (93\%) and EGFR-TKIs in 21 patients (7\%). Patient characteristics are summarized in Table 1.

The median neutrophil count was $7,020 / \mathrm{mm}^{3}$, median lymphocyte count was $1,370 / \mathrm{mm}^{3}$ and median neutrophil to lymphocyte ratio (NLR) was 5.1. Absolute neutrophilia (defined as $\geq 7,500 / \mathrm{mm}^{3}$ in our Institution) was present in 137 patients (44\%), while lymphocytopenia (defined as $<1,500 / \mathrm{mm}^{3}$ in our Institution) was reported in 188 patients $(60 \%)$. Thirty-four patients $(11 \%)$ presented thrombocytosis (defined as $\geq 450,000 / \mathrm{mm}^{3}$ at our Institution).

The best SII cutoff was $\geq 1,270 v s$. $<1,270$, as identified by $\mathrm{X}$-tiles program. Patients were further shared depending on SII into two groups. One hundred and seventy-nine patients had SII $\geq 1,270$ (Group A), whilst 132 had lower SII (Group B).

\section{Overall survival (OS)}

The median OS from first-line therapy was 16.1 months (95\% CI, 13.9 to 18.6 months) in the overall population. One hundred and eighty-nine patients $(61 \%)$ died during their follow-up.

The median OS was 14.4 months (95\% CI, 9.5 to 15.5 months) in males and 19.6 months (95\% CI, 14.4 to 27.9 months) in females $(\mathrm{P}=0.023)$. No significant difference was found between patients aged $<70 v s$. $\geq 70$ years (17.8 vs. 14.4 months, $\mathrm{P}=0.165)$ and between 75 non-smokers patients (19.6 months; $95 \%$ CI, 16.5 to 28.6 months) and 236 smokers (14.6 months; $95 \%$ CI, 10.8 to 16.0 months) $(\mathrm{P}=0.406)$. Otherwise, patients with ECOG-PS $\geq 2$ had a significantly shorter OS compared to ECOG-PS $<2$ (5.0 vs. 16.9 months, $\mathrm{P}=0.002$ ).

Based on histology, the median OS was 12.7 months (95\% CI, 10.3 to 16.3 months) in patients with squamous carcinoma and other histologies and 21.2 months (95\% CI, 16.4 to 27.0 months) in patients with adenocarcinoma $(\mathrm{P}=0.008)$.

Moreover, the median OS was shorter in patients with neutrophilia (11.8 months; $95 \%$ CI, 9.3 to 16.3 months) compared to patients with normal neutrophil count (17.7 months; 95\% CI, 14.4 to 21.4 months) $(\mathrm{P}<0.001)$. On the other hand, no significant differences were found between patients with or without lymphocytopenia (15.0 vs. 16.9 months, $\mathrm{P}=0.207$ ) and with or without thrombocytosis (16.0 vs. 16.1 months, $\mathrm{P}=0.196$ ).

Stratified by SII, the median OS was 12.4 months (95\% CI, 9.4 to 15.7 months) in Group A and 21.7 months (95\% CI, 13.3 to 12.7 months) in Group B $(\mathrm{P}<0.001)$ (Figure 1A). At multivariate analysis, male gender ECOGPS $\geq 2$ and SII $>1,270$ were predictor parameters of worst OS, whilst IV tumor stage was only slightly significant $(\mathrm{P}=0.08)$. Otherwise, only wild-type EGFR status and SII $\geq 1,270$ were independent prognostic factors for worst PFS.

Considering different potential inflammation status in different histological type of NSCLC we calculated SII cut off for patients with adenocarcinomas and with squamous carcinoma, resulting respectively of 1,085 and 562 .

SII was confirmed to be a prognostic factor in patients with adenocarcinomas with a mOS 32,8 months (95\% CI, 27.9 to 37.6 months) in patients with SII $<1,085$ and 19.36 months $(95 \%$ CI, 17.5 to 
Table 1 Patients' characteristics

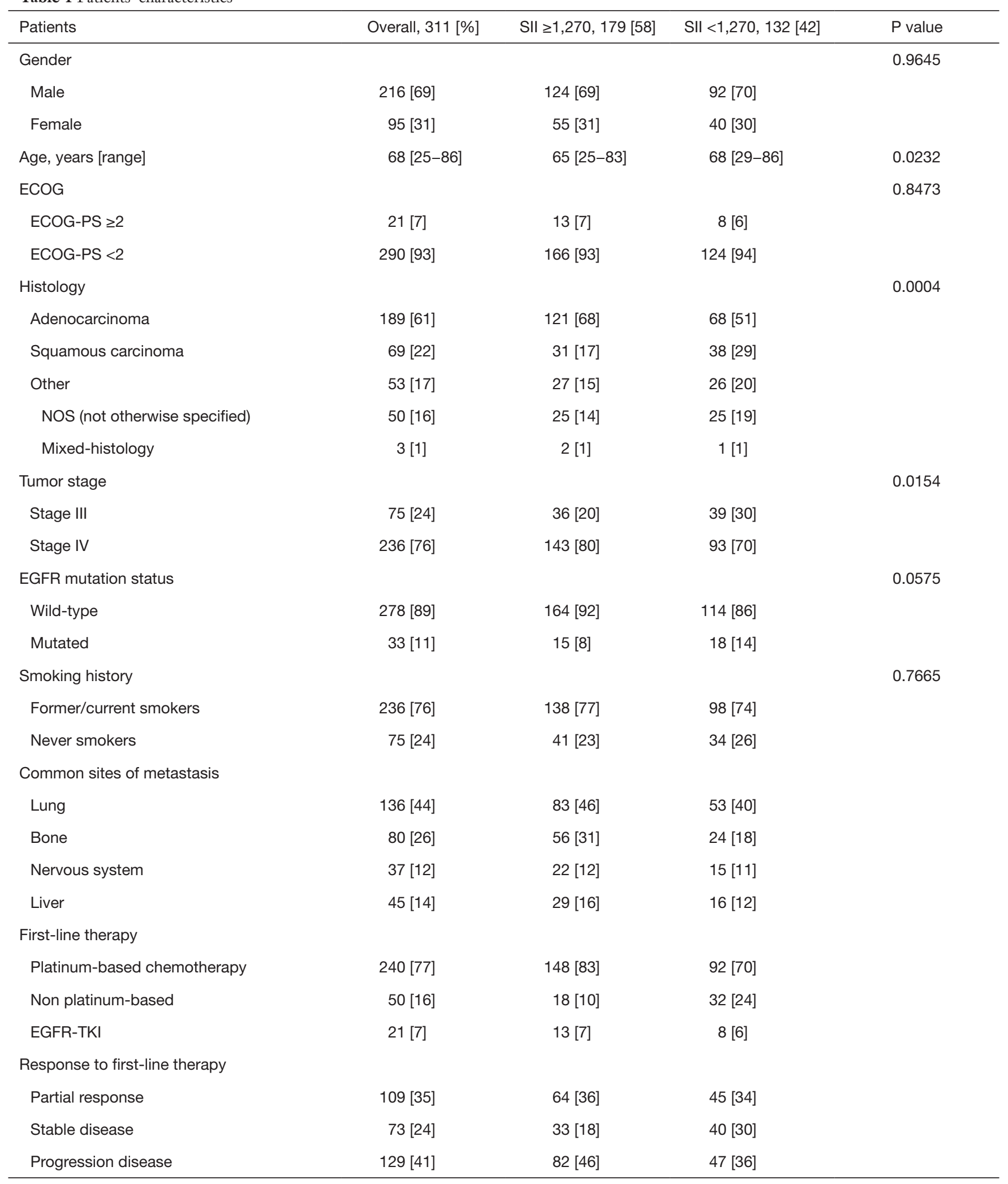



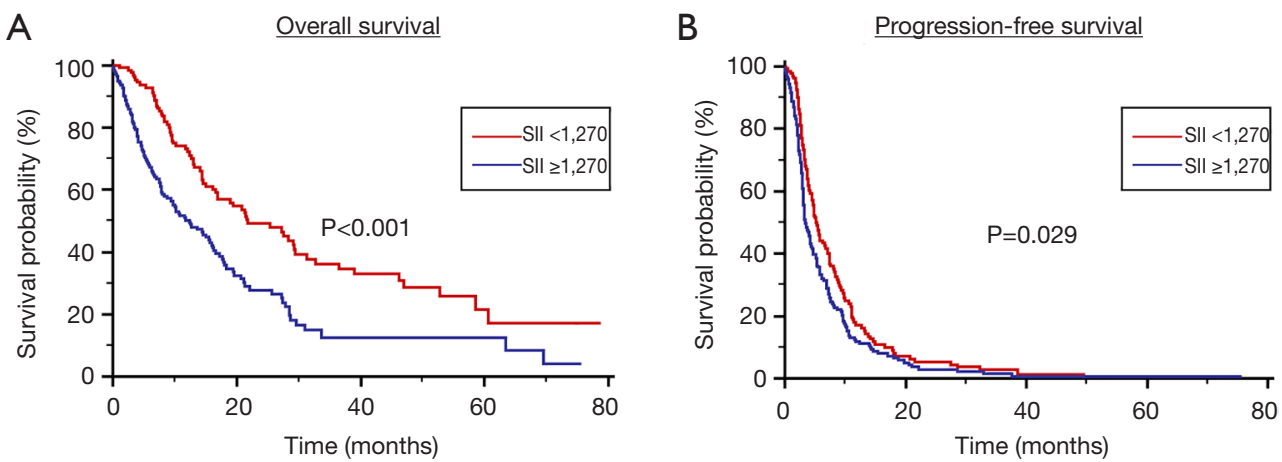

Figure 1 OS (A) and PFS (B) stratified by Systemic Immune-Inflammation Index (SII) in patients treated with first-line therapy for locally advanced or metastatic NSCLC. NSCLC, non-small cell lung cancer; PFS, progression free survival; OS, overall survival.

21.2 months) for those with $\mathrm{SII}>1,085, \mathrm{P}=0.0112$ ) and in patients with squamous carcinoma with a mOS 19.4 months (95\% CI, 14.0 to 24.8 months) in patients with SII $<562$ and 9.5 months (95\% CI, 7.4 to 11.6 months) for those with $\mathrm{SII}>562, \mathrm{P}=0.0219$ ).

\section{Progression-free survival (PFS)}

The median PFS was 4.4 months (95\% CI, 3.9 to 5.1 ) in the overall study population, 5.4 months $(95 \%$ CI, 3.5 to 8.2 months) in the 75 non-smokers and 4.2 months ( $95 \%$ CI, 3.6 to 4.8 months) in the 236 smokers' patients $(\mathrm{P}=0.375)$.

The median PFS was 4.0 months (95\% CI, 3.6 to 4.7 months) in males and 5.4 months (95\% CI, 4.4 to 6.9 months) in females $(\mathrm{P}=0.131)$. No significant difference was found between patients aged $<70 v s . \geq 70$ years (4.2 vs. 4.6 months, $\mathrm{P}=0.568$ ), ECOG-PS $\geq 2$ vs. $<2$ (3.7 vs. 4.2 months, $\mathrm{P}=0.45$ ), histology (squamous carcinoma $v s$. adenocarcinoma vs. other histologies: 3.9 vs. 4.6, respectively).

Furthermore, no differences in terms of median PFS were found between patients with $(3.7$ months; $95 \% \mathrm{CI}$, 3.3 to 4.5 months) and without absolute neutrophilia (4.8 months; $95 \%$ CI, 3.9 to 5.7 months, $\mathrm{P}=0.150$ ). Similarly to OS, no difference was found between patients with and without lymphocytopenia ( 3.5 vs. 4.5 months, $\mathrm{P}=0.379$ ) and with or without thrombocytosis (3.2 vs. 4.4 months, $\mathrm{P}=0.792$ ).

Stratified by SII, the median PFS was 3.3 months (95\% CI, 2.8 to 4.4 months) in Group A and 5.2 months (95\% CI, 4.2 to 7.5 months) in Group B $(\mathrm{P}=0.029)$ (Figure $1 B)$.
Elevated SII was confirmed also to be associated to poorer PFS in patients with adenocarcinomas with a mPFS 10.3 months (95\% CI, 9.6 to 11.0 ) in patients with SII $<1,085$ and 5.8 months (95\% CI, 5.2 to 6.4 ) for those with $\mathrm{SII} \geq 1,085, \mathrm{P}=0.0117$, and in patients with squamous carcinoma with a mPFS 14.6 months (95\% CI, 10.9 to 18.3 months) in patients with SII $<562$ and 3.9 months ( $95 \%$ CI, 2.4 to 5.8 months) for those with $\mathrm{SII} \geq 562, \mathrm{P}=0.0452$.

\section{Univariate and multivariate analyses}

Univariate analysis showed that age ( $\geq 70 v s .<70$ years $)$, male gender, ECOG-PS $\geq 2$, tumor ADC histology, tumor stage IV and SII $\geq 1,270$ were significantly associated with worst OS (Table 2). At multivariate analysis, male gender, ECOG-PS $\geq 2$ and SII $>1,270$ were predictors of worst OS, whilst IV tumor stage was only slightly significant $(\mathrm{P}=0.088)$ (Table 2).

As for PFS, univariate analysis showed that male gender, tumor stage IV, wild-type EGFR status and SII $\geq 1,270$ significantly associated with shorter PFS (Table 3). Multivariate Cox regression analysis revealed that only wild-type EGFR status and SII $\geq 1,270$ were independent prognostic factors for worst PFS (Table 3).

\section{Prognostic models for OS and PFS}

Based on the multivariate analysis, male gender, ECOG-PS $\geq 2$ and SII $>1,270$ were significantly associated with OS.

Patients were stratified according to the absence of the 3 significant prognostic factors (39 patients, $13 \%$ ), presence of 1 (137 patients, $44 \%$ ) or $\geq 2$ factors (135 patients, $43 \%$ ). 
Table 2 Univariate and multivariable analysis of predictors of OS in patients treated with first-line therapy for locally advanced or metastatic NSCLC

\begin{tabular}{|c|c|c|c|c|}
\hline Evaluated variables & \multicolumn{2}{|c|}{ Univariate Cox regression } & \multicolumn{2}{|c|}{ Multivariable cox regression } \\
\hline Age ( $\geq 70$ vs. $<70$ years) & $1.23(0.92-1.63)$ & 0.165 & $1.28(0.95-1.72)$ & 0.113 \\
\hline Gender (F vs. M) & $0.68(0.50-0.95)$ & 0.023 & $0.72(0.51-0.99)$ & 0.047 \\
\hline ECOG-PS ( $\geq 2$ vs. $<2)$ & $2.19(1.35-3.56)$ & 0.002 & $1.84(1.12-3.03)$ & 0.016 \\
\hline Tumor stage (IV vs. III) & $1.46(1.05-2.01)$ & 0.023 & $1.34(0.96-1.86)$ & 0.088 \\
\hline Histology (non-AC vs. AC) & $1.61(1.14-2.47)$ & 0.008 & $0.93(0.58-1.13)$ & 0.074 \\
\hline EGFR status (WT vs. MT) & $0.68(0.37-1.23)$ & 0.204 & & \\
\hline SII $(\geq 1,270$ vs. $<1,270)$ & $1.97(1.46-2.65)$ & $<0.001$ & $1.98(1.47-2.68)$ & $<0.001$ \\
\hline
\end{tabular}

Y, yes; N, no; AC, adenocarcinoma; Cl, confidence interval; ECOG-PS, Eastern Cooperative Oncology Group Performance Status; EGFR, epidermal growth factor receptor; F, female; HR, hazard ratio; M, male; MT, mutated status; NLR, Neutrophil to lymphocyte ratio; WT, wildtype status; NSCLC, non-small cell lung cancer.

Table 3 Univariate and multivariable analysis of predictors of PFS in patients treated with first-line therapy for locally advanced or metastatic NSCLC

\begin{tabular}{lccc}
\hline \multirow{2}{*}{ Evaluated variables } & \multicolumn{2}{c}{ Univariate Cox regression } & \multicolumn{2}{c}{ Multivariable Cox regression } \\
\cline { 2 - 3 } Age $(\geq 70$ vs. $<70$ years $)$ & $1.08(0.85-1.35)$ & P value $(95 \%$ Cl) & 0.568 \\
Gender (F vs. M) & $0.82(0.64-1.06)$ & 0.131 & 1.03 \\
ECOG-PS $(\geq 2$ vs. $<2)$ & $1.18(0.77-1.82)$ & 0.451 & 0.854 \\
Smoke status (Y vs. N) & $1.15(0.85-1.56)$ & 0.377 & 0.99 \\
Tumor stage (IV vs. III) & $1.19(0.93-1.53)$ & 0.168 & 0.987 \\
Histology (non-AC vs. AC) & $1.00(0.82-1.21)$ & 0.983 & 0.54 \\
EGFR status (WT vs. MT) & $0.52(0.34-0.79)$ & 0.002 & 0.006 \\
SII $(\geq 1,270$ vs. $<1,270)$ & $1.32(1.05-1.67)$ & 0.020 & 0.026 \\
\hline
\end{tabular}

Y, yes; N, no; AC, adenocarcinoma; Cl, confidence interval; ECOG-PS, Eastern Cooperative Oncology Group Performance Status; EGFR, epidermal growth factor receptor; F, female; HR, hazard ratio; M, male; MT, mutated status; NLR, neutrophil to lymphocyte ratio; WT, wildtype status; NSCLC, non-small cell lung cancer; PFS, progression free survival.

In the 3 groups, the median OS was 47.0 (95\% CI, 13.0 to 53.9), 19.4 (95\% CI, 5.3 to 23.8) and 9.7 (95\% CI, 3.9 to $12.5)$ months, respectively $(\mathrm{P}<0.001$, Figure $2 A)$.

In the same view, we realized a prognostic model based on the results of the multivariate analysis for PFS. Patients were stratified for PFS based on the presence of 0,1 or 2 factors. The median PFS was 8.7 (95\% CI, 3.0 to 3.9 ), 5.8 (95\% CI, 3.0 to 3.9 ) and 3.3 (95\% CI, 3.0 to 3.9 ) months, respectively $(\mathrm{P}<0.001$, Figure $2 B)$.

\section{Discussion}

Inflammation plays a fundamental role in pathogenesis and tumor progression in patients with NSCLC $(15,16)$. In this view, hematological markers of systemic inflammation should be investigated as predictive and prognostic factors of tumor response. Among them, neutrophils, platelets and lymphocytes seem to be the principal candidate for this role.

Previous studies investigated the prognostic role of NLR, 
A

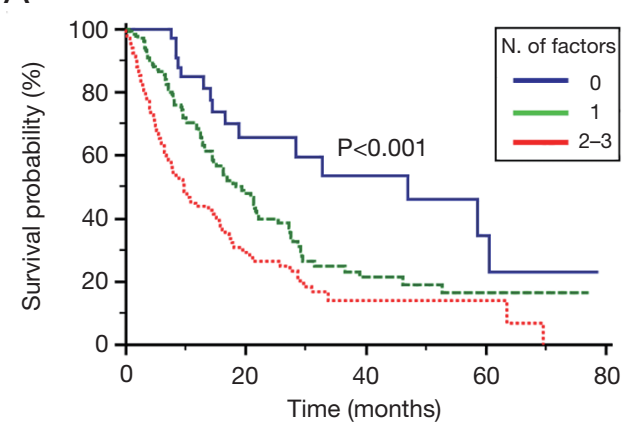

B

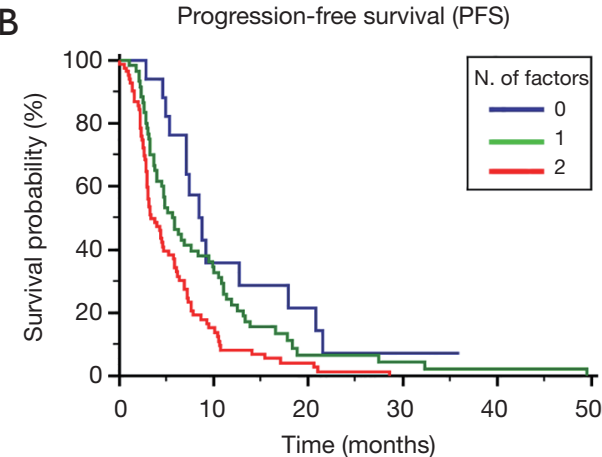

Figure 2 Prognostic models for OS (A) and PFS (B) in patients treated with first-line therapy for locally advanced or metastatic NSCLC. NSCLC, non-small cell lung cancer; PFS, progression free survival; OS, overall survival.

defined as the absolute neutrophil count divided by the absolute lymphocyte count (17-19). Other studies evaluated the platelet to lymphocyte ratio (PLR) (20), defined as the absolute platelets count divided by the absolute lymphocyte count, in NSCLC patients (21). High pretreatment NLR in patients treated with first-line platinum-based chemotherapy was demonstrated to be associated with shorter PFS and OS in advanced NSCLC patients (19). In the same view, high pretreatment PLR was associated to worse response to first-line chemotherapy and to poor prognosis in both non-metastatic and advanced NSCLC patients treated with chemotherapy $(20,21)$.

The prognostic role of platelet count has been investigated in several studies. Recently, Inagaki et al. examined a series of 268 patients with advanced NSCLC and they reported that a platelet volume/platelet count (MPV/PC) ratio lower than 0.41 was associated with a worst prognosis (22). Similarly, the presence of preoperative thrombocytosis has been correlated with shorter OS and DFS in patients with resectable NSCLC (5), as well as with higher mortality and resistance to cisplatin-based chemotherapy in locally advanced and metastatic patients with lung cancer (23).

Recently, the negative prognostic role of high platelets/ lymphocyte ratio (PLR) has been evaluated in this setting. Indeed, high PLR was associated with poor prognosis in patients with early-stage NSCLC treated with stereotactic radiation (24). In the same view, high PLR was a negative independent risk factor to first-line platinum-based chemotherapy in patients with advanced NSCLC (20).

Based on the recent results regarding the use of antiprogrammed death-1 (PD-1)/PD-ligand 1 (PD-L1) agents nivolumab (25-27) and pembrolizumab $(28,29)$, the research for reliable inflammatory biomarkers will be a major focus in future years. Among them, the SII, a novel immune inflammation-based prognostic score that combines neutrophil, lymphocyte and platelet counts, seems to merit fully consideration. In fact, several studies demonstrated that other inflammatory indexes analyzing the same parameters, such as NLR and PLR, represents prognostic factors for patients receiving immunotherapy (30). In our study, we assessed the prognostic role of SII, a novel immune inflammation-based prognostic score that combines neutrophil, lymphocyte and platelet counts. We showed that a SII higher than 1,270 is associated with a shorter OS and PFS in patients with locally advanced or metastatic NSCLC treated with firstline chemo- or targeted therapies. Moreover, SII resulted a significant independent prognostic factor for both OS and PFS at univariate and multivariate analyses.

Based on these results, we designed two different prognostic models for OS and PFS. Regarding OS, a model based on the presence of ECOG-PS $\geq 2$, IV tumor stage and SII $>1,270$ has allowed identifying 3 different groups of prognostic risk. As for PFS, EGFR status and SII $>1,270$ permitted to stratify patients into good, intermediate and poor risk categories. The higher cut off-value in our study $[1,270]$, compared to the cut off-value identified by $\mathrm{Hu}$ and his group [330] may be explained by the major frequency of thrombocytopenia in patients with cirrhosis and HCC (31).

However, there are some limitations to this study, due firstly to its retrospective nature, which is susceptible to bias in data selection and analysis. In addition, other inflammatory markers, including CRP (C reactive protein) or procalcitonin, are not routinely measured at our institutions. Furthermore, SII can be influenced by concurrent infection and drugs that cannot be accounted in this study. 


\section{Conclusions}

In conclusion, SII seems to have a significant prognostic impact in patients with locally advanced or metastatic NSCLC. These observations should be considered relevant for the therapeutic strategy and management of such patients. Further studies in larger populations should be conducted to validate the prognostic role of SII and our risk models in clinical practice.

\section{Acknowledgments}

The study was realized with Authors' University Funding (Università Politecnica Marche, Ancona, Italy).

\section{Footnote}

Conflicts of Interest: The authors have no conflicts of interest to declare.

Ethical Statement: The authors are accountable for all aspects of the work in ensuring that questions related to the accuracy or integrity of any part of the work are appropriately investigated and resolved. Our project has been approved by local Ethics Committee (ID 2019-146) and the work conforms to the provisions of in accordance with the Helsinki Declaration as revised in 2013.

\section{References}

1. Jemal A, Bray F, Center MM, et al. Global cancer statistics. CA Cancer J Clin 2011;61:69-90.

2. Skillrud DM, Offord KP, Miller RD. Higher risk of lung cancer in chronic obstructive pulmonary disease. A prospective, matched, controlled study. Ann Intern Med 1986;105:503-7.

3. Baron JA, Sandler RS. Nonsteroidal anti-inflammatory drugs and cancer prevention. Annu Rev Med 2000;51:511-23.

4. Teramukai S, Kitano T, Kishida Y, et al. Pretreatment neutrophil count as an independent prognostic factor in advanced non-small-cell lung cancer: an analysis of Japan Multinational Trial Organisation LC00-03. Eur J Cancer 2009;45:1950-8.

5. Kim M, Chang H, Yang HC, et al. Preoperative thrombocytosis is a significant unfavorable prognostic factor for patients with resectable non-small cell lung cancer. World J Surg Oncol 2014;12:37.
6. Kim SH, Lee HW, Go SI, et al. Clinical significance of the preoperative platelet count and platelet-to-lymphocyte ratio (PLT-PLR) in patients with surgically resected nonsmall cell lung cancer. Oncotarget 2016;7:36198-206.

7. Shimizu K, Okita R, Saisho S, et al. Preoperative neutrophil/lymphocyte ratio and prognostic nutritional index predict survival in patients with non-small cell lung cancer. World J Surg Oncol 2015;13:291.

8. Kim KH, Park TY, Lee JY, et al. Prognostic significance of initial platelet counts and fibrinogen level in advanced non-small cell lung cancer. J Korean Med Sci 2014;29:507-11.

9. Badovinac S, Korsic M, Mursic D, et al. Cancer-related inflammation as predicting tool for treatment outcome in locally advanced and metastatic non-small cell lung cancer. J Thorac Dis 2016;8:1497-503.

10. Hu B, Yang XR, Xu Y, et al. Systemic immuneinflammation index predicts prognosis of patients after curative resection for hepatocellular carcinoma. Clin Cancer Res 2014;20:6212-22.

11. Therasse P, Arbuck SG, Eisenhauer EA, et al. New guidelines to evaluate the response to treatment in solid tumors. European Organization for Research and Treatment of Cancer, National Cancer Institute of the United States, National Cancer Institute of Canada. J Natl Cancer Inst 2000;92:205-16.

12. Camp RL, Dolled-Filhart M, Rimm DL. X-tile: a new bio-informatics tool for biomarker assessment and outcome-based cut-point optimization. Clin Cancer Res 2004;10:7252-9.

13. Fagerland MW, Hosmer DW, Bofin AM. Multinomial goodness-of-fit tests for logistic regression models. Stat Med 2008;27:4238-53.

14. Mickey RM, Greenland S. The impact of confounder selection criteria on effect estimation. Am J Epidemiol 1989;129:125-37.

15. Gomes M, Teixeira AL, Coelho A, et al. The role of inflammation in lung cancer. Adv Exp Med Biol 2014;816:1-23.

16. Yildirim M, Yildiz M, Duman E, et al. Prognostic importance of the nutritional status and systemic inflammatory response in non-small cell lung cancer. J BUON 2013;18:728-32.

17. Cedrés S, Torrejon D, Martínez A. Neutrophil to lymphocyte ratio (NLR) as an indicator of poor prognosis in stage IV non-small cell lung cancer. Clin Transl Oncol 2012;14:864-9.

18. Yao Y, Yuan D, Liu H, et al. Pretreatment neutrophil to 
lymphocyte ratio is associated with response to therapy and prognosis of advanced non-small cell lung cancer patients treated with first-line platinum-based chemotherapy. Cancer Immunol Immunother 2013;62:471-9.

19. Berardi R, Rinaldi S, Santoni M, et al. Prognostic models to predict survival in patients with advanced non-small cell lung cancer treated with first-line chemo- or targeted therapy. Oncotarget 2016;7:26916-24.

20. Liu H, Wu Y, Wang Z, et al. Pretreatment platelet-tolymphocyte ratio (PLR) as a predictor of response to firstline platinum-based chemotherapy and prognosis for patients with non-small cell lung cancer. J Thorac Dis 2013;5:783-9.

21. Unal D, Eroglu C, Kurtul N, et al. Are neutrophil/ lymphocyte and platelet/lymphocyte rates in patients with non-small cell lung cancer associated with treatment response and prognosis? Asian Pac J Cancer Prev 2013;14:5237-42.

22. Inagaki N, Kibata K, Tamaki T, et al. Prognostic impact of the mean platelet volume/platelet count ratio in terms of survival in advanced non-small cell lung cancer. Lung Cancer 2014;83:97-101.

23. Du G, Yang Y, Zhang Y, et al. Thrombocytosis and immunohistochemical expression of connexin 43 at diagnosis predict survival in advanced non-small-cell lung cancer treated with cisplatin-based chemotherapy. Cancer Chemother Pharmacol 2013;71:893-904.

24. Cannon NA, Iyengar P, Choy H, et al. Stereotactic ablative body radiation therapy for tumors in the lung

Cite this article as: Berardi $\mathrm{R}$, Santoni $\mathrm{M}$, Rinaldi S, Bower M, Tiberi M, Morgese F, Caramanti M, Savini A, Ferrini C, Torniai M, Fiordoliva I, Newsom-Davis T. Pre-treatment systemic immune-inflammation represents a prognostic factor in patients with advanced non-small cell lung cancer. Ann Transl Med 2019;7(20):572. doi: 10.21037/atm.2019.09.18 in octogenarians: a retrospective single institution study. BMC Cancer 2014;14:971.

25. Gettinger SN, Horn L, Gandhi L, et al. Overall Survival and Long-Term Safety of Nivolumab (Anti-Programmed Death 1 Antibody, BMS-936558, ONO-4538) in Patients With Previously Treated Advanced Non-Small-Cell Lung Cancer. J Clin Oncol 2015;33:2004-12.

26. Borghaei H, Paz-Ares L, Horn L, et al. Nivolumab versus Docetaxel in Advanced Nonsquamous Non-Small-Cell Lung Cancer. N Engl J Med 2015;373:1627-39.

27. Brahmer J, Reckamp KL, Baas P, et al. Nivolumab versus Docetaxel in Advanced Squamous-Cell Non-Small-Cell Lung Cancer. N Engl J Med 2015;373:123-35.

28. Herbst RS, Baas P, Kim DW, et al. Pembrolizumab versus docetaxel for previously treated, PD-L1-positive, advanced non-small-cell lung cancer (KEYNOTE-010): a randomised controlled trial. Lancet 2016;387:1540-50.

29. Garon EB, Rizvi NA, Hui R, et al. Pembrolizumab for the Treatment of Non-Small-Cell Lung Cancer. N Engl J Med 2015;372:2018-28.

30. Diem S, Schmid S, Krapf M, et al. Neutrophil-toLymphocyte ratio (NLR) and Platelet-to-Lymphocyte ratio (PLR) as prognostic markers in patients with nonsmall cell lung cancer (NSCLC) treated with nivolumab. Lung Cancer 2017;111:176-81.

31. Lu SN, Wang JH, Liu SL, et al. Thrombocytopenia as a surrogate for cirrhosis and a marker for the identification of patients at high-risk for hepatocellular carcinoma. Cancer 2006;107:2212-22. 